\title{
Effects of Equipment-Structure-Soil Interaction on Seismic Response of Equipment and Structure via Real-Time Dynamic Substructuring Shaking Table Testing
}

\author{
Chongxiang Zhang ${ }^{1,2}$ and Nan Jiang ${ }^{1,2}$ \\ ${ }^{1}$ School of Civil Engineering, Tianjin University, Tianjin 300072, China \\ ${ }^{2}$ Key Laboratory of Coastal Civil Engineering Structure and Safety, Tianjin University, Ministry of Education, Tianjin 300072, China \\ Correspondence should be addressed to Nan Jiang; jiangnan@tju.edu.cn
}

Received 25 April 2017; Revised 23 August 2017; Accepted 18 September 2017; Published 15 November 2017

Academic Editor: Mahmoud Bayat

Copyright (c) 2017 Chongxiang Zhang and Nan Jiang. This is an open access article distributed under the Creative Commons Attribution License, which permits unrestricted use, distribution, and reproduction in any medium, provided the original work is properly cited.

\begin{abstract}
Equation of motion for an equipment-structure-soil (ESS) interaction system was derived using the branch substructure method. After rearrangement, this equation was applied to real-time dynamic substructuring shaking table (RTDSST) testing of the ESS system. This method adopts the equipment-structure (ES) subsystem as the experimental substructure and a modal reduced soil model as the numerical substructure: the former is tested via the shaking table, and the latter is numerically simulated, while realtime data communication occurs between the two substructures during testing. A scale model of the ESS system was designed and underwent an RTDSST test. The experimental data were found to be consistent with the numerical calculation results, which corroborates the reliability and validity of the proposed testing method. A comparison of the experimental results from different earthquake stages implies that seismic responses of the equipment and structure decreased in general due to the intervention of soil, but the soil effect weakened with earthquake intensity.
\end{abstract}

\section{Introduction}

With increasing number of nonstructural members being incorporated into modern building systems, damage to precision equipment and other nonstructural members may result in huge economic losses and serious safety hazards. Recent research has shown that disregard of equipmentstructure interaction in some situations has an adverse impact on the seismic design of structures and equipment $[1,2]$. Most studies of the ES subsystem were based on the rigid foundation assumption with no account taken of the soil effect, which is at odds with engineering reality and impairs the seismic performance research validity. The soil-structure interaction problem had also been investigated by using the shaking table test [3-5] and numerical analysis of soilstructure interaction [6-8]. These studies treated soil and structure as a fully coupled interaction system to elucidate the interaction effects.
Jiang et al. $[9,10]$ performed shaking table testing with an account of soil-structure interaction, which was found to have a strong effect on the upper structure response. The soil contribution can prolong the period of vibration of the entire model, deteriorate the upper structure actual seismic performance, and even introduce errors into the calculation results. Therefore, the overall ESS interaction system performance requires the experimental corroboration. However, in the conventional shaking table testing, construction of the entire ESS system would be too complicated and expensive. This necessitates the development of a new test method that would improve the efficiency and accuracy of such testing and provide a more reliable basis for the seismic design of structures and equipment.

The implementation of such large-scale complex tests became possible with the development of the following approach. In 1992, Nakashima et al. [11] introduced the real-time dynamic substructuring (RTDS) test method. This 
is a method of dynamically testing a structure without experimentally testing a physical model of the entire system. Instead, the structure can be split into two coupled parts, the region of particular interest, which is tested experimentally, and the remainder, which is tested numerically. The force and displacement at the interface of numerical and physical substructures are transferred via a transfer system using a servo-hydraulic actuator or a shaking table.

This method can effectively reduce the model scale and testing difficulties. In the RTDSST tests with an account of the soil effect [12-14], the soil and upper structure are mostly treated as the numerical and experimental substructures, respectively, while the dynamic response of the overall coupled system investigation is based on the real-time data communication between the substructures. Given the realtime requirement of testing, calculations of the numerical substructure are often reformed via simplified models, such as the lumped parameter soil model proposed by Wang et al. [12], the modified Penzien model adopted by Yan et al. [13], and the simplified single-degree-of-freedom (DOF) model introduced by Tang et al. [14]. The use of simplified computational models can ensure efficient calculation of numerical substructure in substructure testing but may hurt the accuracy of calculation when applied to complex soil models. Moreover, the direct application of complex finite element models does not allow one to implement real-time substructure testing. Jiang et al. $[15,16]$ employed a branch substructure method in an in-depth study of soil-structure interaction, wherein the calculation time was substantially reduced by the soil modal reduction. The motion equation of equip-structure-soil dynamic interaction system can be deduced using the method of branch substructure. After rearrangement, this equation was applied to real-time dynamic substructuring shaking table test.

In this study, the ESS interaction system equation of motion was derived using the branch substructure method presented elsewhere in the literature [15]. The equation was then transformed and applied to the RTDSST testing of an ESS system. This testing method adopts the ES subsystem as the experimental substructure and the soil as the numerical substructure. The former is subjected to loading and control imposed by the shaking table, while the latter is numerically simulated using a respective software program. A scale model of the ESS interaction system was designed, where the equipment was reduced to a single-DOF model and the structure was modeled as a four-story steel frame. An experimental system was constructed to perform an RTDSST test on the model, to study the behavior of the ESS interaction system under seismic excitations. After the reliability of the testing method proposed in this study was verified, a comparative analysis of the seismic performance of the equipment and structure under the soil effect was performed.

\section{The RTDSST Testing Method Based on the Branch Substructure Method}

An ESS interaction system consists of three components: equipment, structure, and soil. The testing method proposed in this study treats the ES subsystem as the experimental

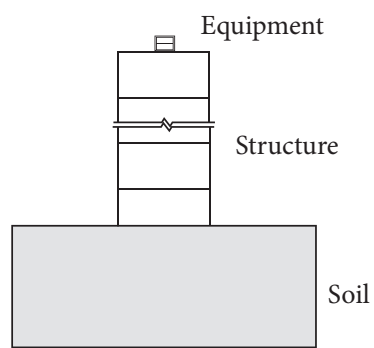

(a) Equipment-structure-soil interaction system

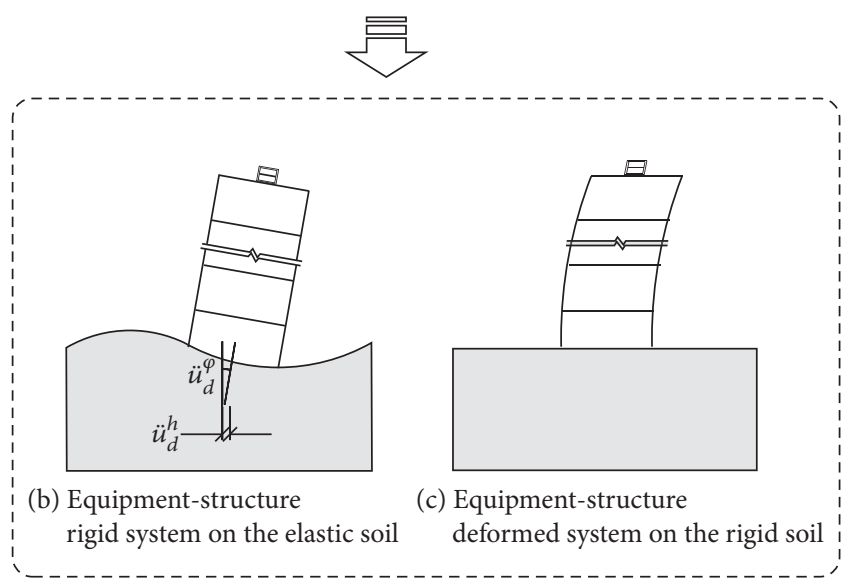

FIgURE 1: Schematic for motion equation of the ESS interaction system.

substructure and the soil as the numerical substructure. The former is subjected to the loading and control applied by the shaking table, while the latter is computed and analyzed by the simulation software. Real-time data communication occurs between the two substructures during testing. Based on the branch substructure method, the ESS interaction system is subdivided into branch $d$ corresponding to the rigid ES system on elastic soil and branch $s$ corresponding to the deformed ES system on rigid soil, as is shown in Figures 1(b) and $1(\mathrm{c})$, respectively.

The modal transformation matrix consisting of $m$ th order modes for branch $d$, denoted by $\Phi_{d}$, can be obtained as follows:

$$
\begin{aligned}
{\left[k_{d}\right]\left\{\phi_{d}\right\} } & =\lambda_{d}\left[m_{d}\right]\left\{\phi_{d}\right\} \\
{\left[\Phi_{d}\right] } & =\left[\{\phi\}_{d}^{1} \cdots\{\phi\}_{d}^{i} \cdots\{\phi\}_{d}^{m}\right],
\end{aligned}
$$

where $k_{d}$ and $m_{d}$ are stiffness and mass matrices of branch $d$, respectively. The calculation matrices after modal transformation of branch $d$ are as follows:

$$
\begin{aligned}
\left\{u_{d}\right\} & =\left[\Phi_{d}\right]\left\{q_{d}\right\} \\
{\left[\widetilde{k}_{d}\right] } & =\left[\Phi_{d}\right]^{T}\left[k_{d}\right]\left[\Phi_{d}\right] \\
{\left[\widetilde{m}_{d}\right] } & =\left[\Phi_{d}\right]^{T}\left[m_{d}\right]\left[\Phi_{d}\right] \\
{\left[\widetilde{c}_{d}\right] } & =\left[\Phi_{d}\right]^{T}\left[c_{d}\right]\left[\Phi_{d}\right] \\
{\left[\tilde{f}_{d}\right] } & =\left[\Phi_{d}\right]^{T}\left[f_{d}\right],
\end{aligned}
$$


where $u_{d}$ and $q_{d}$ are physical displacements and modal coordinates of branch $d$, respectively; $c_{d}$ denotes the damping matrix of branch $d$, which can be calculated using the general damping theory; and $f_{d}$ is the load matrix of branch $d$. As the ES subsystem has a small number of DOFs, there is no need for modal reduction, and the complete calculation matrices are retained. The displacement of the ES subsystem, $u_{s}$, is composed of two parts, rigid-body displacement of the ES subsystem (Figure 1(b)) caused by soil displacement and the intrinsic displacement of the subsystem $q_{s}$ (Figure 1(c)), and can be written in the following form:

$$
\left\{u_{s}\right\}=[R]\left[\Phi_{d}\right]\left\{q_{d}\right\}+\left\{q_{s}\right\},
$$

where $R$ can be calculated from the rigid-body displacement of the ES subsystem induced by the deformation of soil in branch $d$. The relationship between the displacements of the ES subsystem and soil can be expressed in the matrix form:

$$
\left\{\begin{array}{l}
u_{d} \\
u_{s}
\end{array}\right\}=\left[\begin{array}{cc}
\Phi_{d} & 0 \\
R \Phi_{d} & I
\end{array}\right]\left\{\begin{array}{l}
q_{d} \\
q_{s}
\end{array}\right\} .
$$

Combining and rearranging the above equations by the coordinate relationship of deformation gives the equation of motion for the entire ESS interaction system:

$$
\begin{gathered}
{\left[\begin{array}{cc}
\widetilde{m}_{d} & \Phi_{d}^{T} R^{T} m_{s} \\
m_{s} R \Phi_{d} & m_{s}
\end{array}\right]\left\{\begin{array}{l}
\ddot{q}_{d} \\
\ddot{q}_{s}
\end{array}\right\}+\left[\begin{array}{cc}
\tilde{c}_{d} & 0 \\
0 & c_{s}
\end{array}\right]\left\{\begin{array}{c}
\dot{q}_{d} \\
\dot{q}_{s}
\end{array}\right\}} \\
+\left[\begin{array}{cc}
\tilde{k}_{d} & 0 \\
0 & k_{s}
\end{array}\right]\left\{\begin{array}{l}
q_{d} \\
q_{s}
\end{array}\right\}=\left\{\begin{array}{l}
\tilde{f}_{d} \\
f_{s}
\end{array}\right\},
\end{gathered}
$$

where $m_{s}, c_{s}, k_{s}$, and $f_{s}$ are the mass, damping, stiffness, and load matrices of the ES subsystem in branch $s$, respectively. The coupling term $m_{s} R \Phi_{d}$ in the equation above acts as a link between the ES subsystem and soil. By rearranging the coupling-related terms to the right-hand side of this equation, the equations of motion of the soil and the ES subsystem are obtained. These equations are as follows:

$$
\begin{aligned}
\widetilde{m}_{d} \ddot{q}_{d}+\widetilde{c}_{d} \dot{q}_{d}+\tilde{k}_{d} q_{d} & =\tilde{f}_{d}-\Phi_{d}^{T} R^{T} m_{s} \ddot{q}_{s} \\
m_{s} \ddot{q}_{s}+c_{s} \dot{q}_{s}+k_{s} q_{s} & =f_{s}-m_{s} R \Phi_{d} \ddot{q}_{d} .
\end{aligned}
$$

In the shaking table testing substructure used in this study, the equipment in the ESS system was simulated with a single-DOF model, and the four-story steel frame was reduced to a shear model with four DOFs. Thus, the equipment and structure formed a computational model with five DOFs, so $R$ can be presented as a $5 \times n$ matrix ( $n$ is the total number of DOFs of the soil) as follows:

$$
R=\left[\begin{array}{ccccc}
1 & h_{1} & 0 & \cdots & 0 \\
1 & h_{2} & 0 & \cdots & 0 \\
1 & h_{3} & 0 & \cdots & 0 \\
1 & h_{4} & 0 & \cdots & 0 \\
1 & h_{5} & 0 & \cdots & 0
\end{array}\right] .
$$

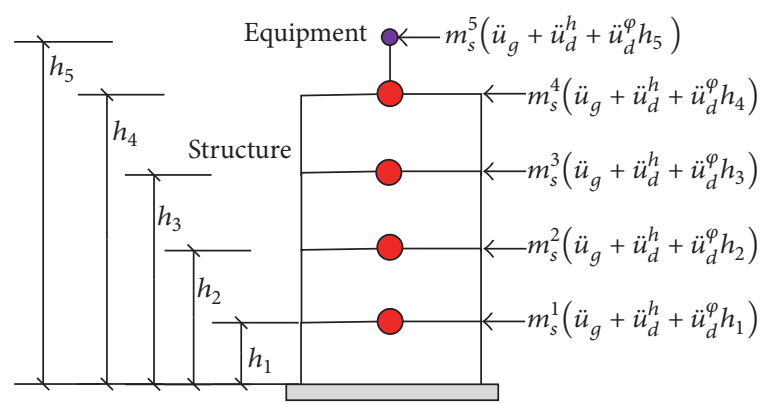

Figure 2: Equivalent load transformation.

As seen from Figure 2, which depicts the external and coupling loads on the right-hand side of the equation of motion for the ES subsystem in (7), the effect of foundation rotational acceleration, $\ddot{u}_{d}^{\varphi}$, on each DOF correlates with height. To realize the foundation rotational acceleration in the horizontal shaking table test, $\ddot{u}_{d}^{\varphi}$ is converted into an equivalent horizontal load based on the principle of equivalence. The respective conversion is given by

$$
\begin{aligned}
& m_{s}^{1}\left(\ddot{u}_{g}+\ddot{u}_{d}^{h}+\ddot{u}_{d}^{\varphi} h_{1}\right)+m_{s}^{2}\left(\ddot{u}_{g}+\ddot{u}_{d}^{h}+\ddot{u}_{d}^{\varphi} h_{2}\right) \\
& \quad+m_{s}^{3}\left(\ddot{u}_{g}+\ddot{u}_{d}^{h}+\ddot{u}_{d}^{\varphi} h_{3}\right)+m_{s}^{4}\left(\ddot{u}_{g}+\ddot{u}_{d}^{h}+\ddot{u}_{d}^{\varphi} h_{4}\right) \\
& \quad+m_{s}^{5}\left(\ddot{u}_{g}+\ddot{u}_{d}^{h}+\ddot{u}_{d}^{\varphi} h_{5}\right)=m_{s}^{1} \ddot{u}_{\mathrm{eq}}+m_{s}^{2} \ddot{u}_{\mathrm{eq}}+m_{s}^{3} \ddot{u}_{\mathrm{eq}} \\
& \quad+m_{s}^{4} \ddot{u}_{\mathrm{eq}}+m_{s}^{5} \ddot{u}_{\mathrm{eq}} \\
& \ddot{u}_{\mathrm{eq}}=\ddot{u}_{g}+\ddot{u}_{d}^{h} \\
& \quad+\frac{m_{s}^{1} h_{1}+m_{s}^{2} h_{1}+m_{s}^{3} h_{1}+m_{s}^{4} h_{1}+m_{s}^{5} h_{5}}{m_{s}^{1}+m_{s}^{2}+m_{s}^{3}+m_{s}^{4}+m_{s}^{5}} \ddot{u}_{d}^{\varphi}
\end{aligned}
$$

The procedure of the RTSST testing of the ESS interaction system is as follows:

(a) Assume that the external load and coupling load acting on the soil at the $i$ th time step are known.

(b) Calculate the soil acceleration response at the $(i+$ $\Delta t$ )th time step.

(c) Obtain the external and coupling loads acting on the ES subsystem.

(d) Calculate the equivalent horizontal acceleration based on the principle of equivalence and use the results as a new input data to control the shaking table and, thus, apply the equivalent load to the experimental substructure.

(e) Calculate the force generated by the experimental substructure from the measured acceleration of the ES subsystem and seismic excitations and then transfer this force to the soil for use in the next numerical simulation. In this way, the interactive communication of experimental and numerical data between the shaking table and simulation software is realized until the test termination. 


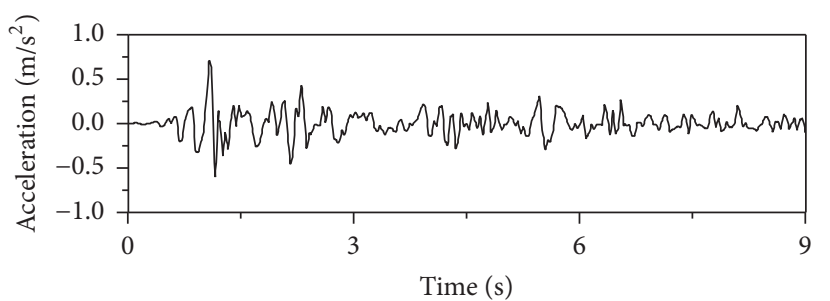

(a) El Centro

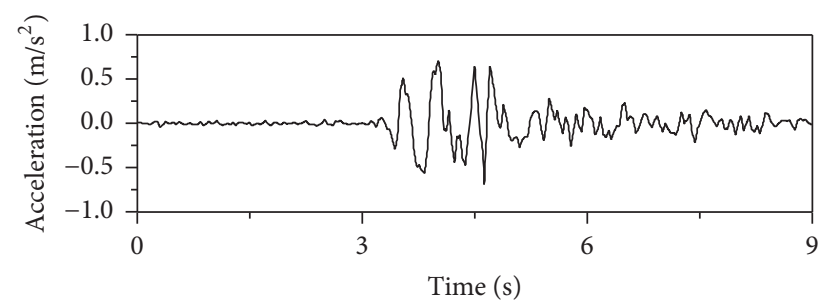

(b) Tianjin

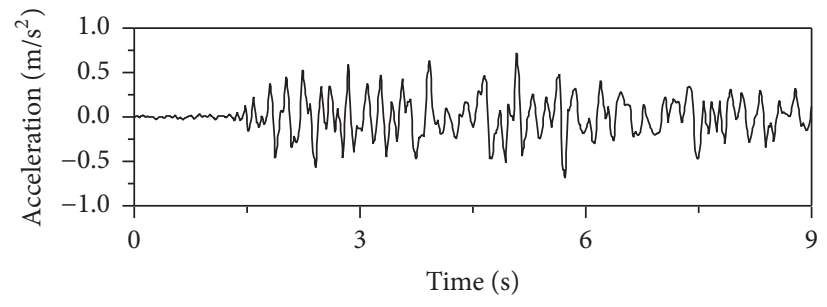

(c) PerSon

Figure 3: Acceleration time history curves of three ground motions.

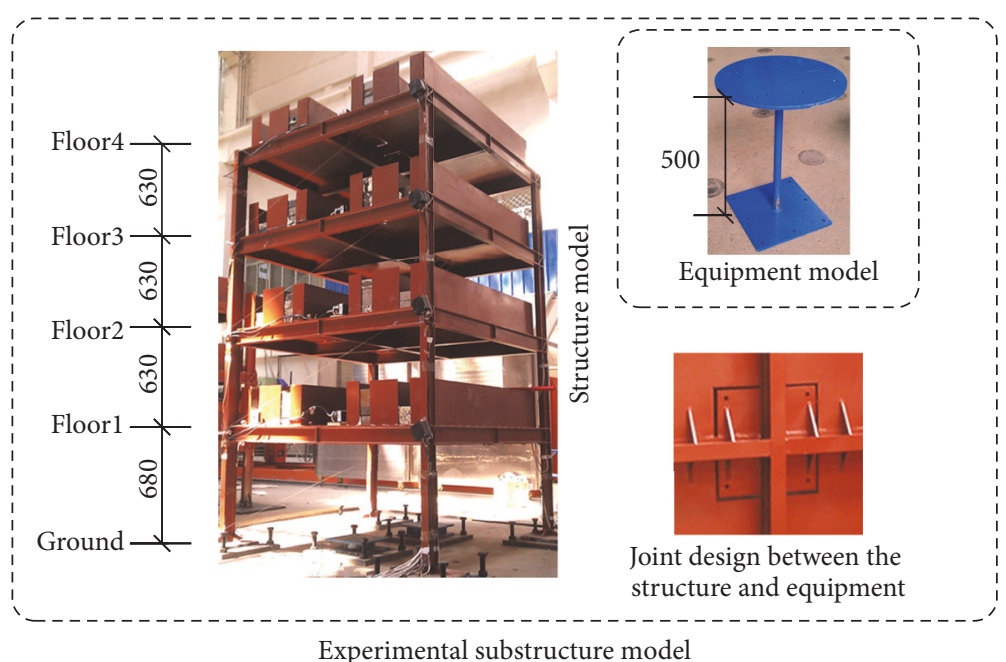

FIGURE 4: Model of the experimental substructure.

The stiffness matrix of equipment-structure-soil motion equation will be decoupled using the branch substructure method so that the motion equation can be directly applied to a shaking table real-time substructure experiment. The branch substructure method also provides a suitable solution for other complex interaction systems.

\section{Simulation and Test Procedures}

A one-fifth scale model of an ESS interaction system was elaborated in this study. In this model, the equipment was a single-DOF model built on the top of the four-story steel frame structure. The ES subsystem was used as the experimental substructure, which would be subjected to loading and control imposed by the shaking table during testing. The soil was modeled as a type III site, and it was adopted as a numerical substructure in the RTDSST testing. Because the finite element model of soil which contained too many DOFs may not satisfy the real-time requirement of shaking table substructure experiment. The Ritz vector method was used to reduce DOFs. The testing was performed on a $3 \mathrm{~m}$ $\times 3 \mathrm{~m}$ shaking table at the Beijing University of Technology. The El Centro, TianJin, and PerSon ground motions, which represent different loading patterns applicable for type III sites, were selected as the seismic inputs and the respective acceleration time history curves (Figure 3). The El Centro and TianJin ground motions were seismic records waves, while the PerSon ground motion was an artificial seismic wave. The strain, acceleration, and displacement responses of the total system were measured during testing.

3.1. Equipment-Structure Model. The structure of the ES subsystem model in Figure 4 consisted of $\mathrm{H}$-beam steel sections. The first story of the structure was $0.68 \mathrm{~m}$ high, 
TABLE 1: Material parameters of the soil and foundation model.

\begin{tabular}{|c|c|c|c|c|c|}
\hline Type & Depth $(\mathrm{m})$ & Elastic modulus $(\mathrm{Pa})$ & Density $\left(\mathrm{kg} / \mathrm{m}^{3}\right)$ & Viscous force $(\mathrm{Pa})$ & Friction angle $\left(^{\circ}\right)$ \\
\hline Foundation & 0.4 & $3.52 \times 10^{10}$ & 2650 & - & - \\
\hline Soil layer 1 & 3.6 & $2.11 \times 10^{7}$ & 1730 & $1.27 \times 10^{5}$ & 15.3 \\
\hline Soil layer 2 & 8.4 & $5.64 \times 10^{7}$ & 1950 & $1.65 \times 10^{5}$ & 16.8 \\
\hline Soil layer 3 & 3.0 & $3.37 \times 10^{8}$ & 2030 & $1.84 \times 10^{5}$ & 21.6 \\
\hline
\end{tabular}

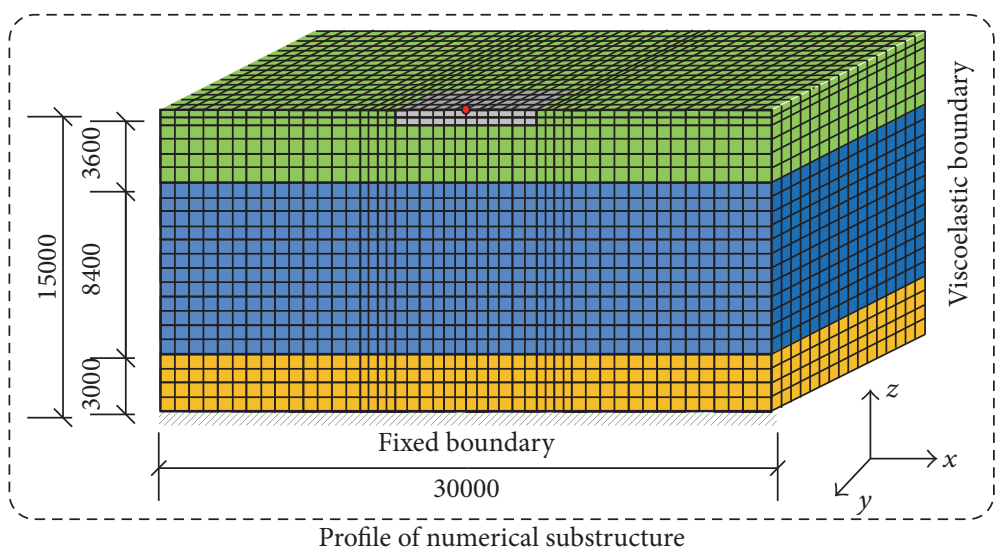

Figure 5: Model of the numerical substructure.

and each of the other stories was $0.63 \mathrm{~m}$ high. The structure length and width were both equal to $1.6 \mathrm{~m}$. Each of the lower three stories weighed $1,700 \mathrm{~kg}$, while the fourth story weighed $1,540 \mathrm{~kg}$. The equipment in the model consisted of circular steel pipes and had a height of $0.5 \mathrm{~m}$ and a weight of $90 \mathrm{~kg}$. The elastic modulus and yield strength of the H-beam steel sections were $202.0 \mathrm{GPa}$ and $339.6 \mathrm{MPa}$, respectively, while those of the circular steel pipes were 192.0 GPa and $421.4 \mathrm{MPa}$, respectively. A steel plate was attached to the center of the structure top, while the equipment was anchored to the plate using anchor bolts during testing.

3.2. Soil Model. Table 1 shows the material parameters of the soil model. The overall dimensions of the soil model were $30 \mathrm{~m} \times 15 \mathrm{~m} \times 15 \mathrm{~m}$, whereas the foundation embedded in it had dimensions of $2.2 \mathrm{~m} \times 2.2 \mathrm{~m} \times 0.4 \mathrm{~m}$. A soil finite element model (FEM) (Figure 5) was developed using the ANSYS commercial finite element software by the experimental design requirements [17]. An eight-node solid element was used to model the soil, with each node having three DOFs, that is, translation along the $x, y$, and $z$ directions. The element discretization should satisfy the relationship $h_{\max }=$ (0.200 0.125) $\lambda_{s}$, where $\lambda_{s}$ is the wavelength, which ensures that the element width does not exceed fivefold values of the element height. The motion of the experimental substructure model is dominated by translation along the $x$-axis, and the soil primarily underwent translational displacement along the $x$-axis. Therefore, a constraint equation of the FEM was applied for the problem simplification. Regarding boundary conditions, the soil bottom was fixed, while its other sides were modeled by viscoelastic boundaries [18].

As the soil FEM had a large number of DOFs, it could not be directly used in the RTDSST testing. Therefore, modal reduction of the soil was performed. First, calculation matrices for the soil were exported via the ANSYS software command stream and then imported into MATLAB for the modal reduction. Given the discrete distribution of the dominant soil mode, Li and Jiang [19] analyzed the relationship between the external load on soil and its vibration behavior and calculated the soil modal vector using the Ritz vector method. It was found that this method could tune off the vibration modes that had a relatively feeble effect on the soil vibration. In the current study, this method was employed for the realization of the Ritz vector analysis with the soil vibration mode and frequency assessment at each order. Table 2 compares the calculation results with those from the complete modal model of soil. The standard requires the total ratio of participating mass to exceed $90 \%$. The mass participation factors for various orders of the Ritz vectors were calculated for the soil. According to the results obtained, the sum of the mass participation factors for the 1st through 60 th orders reached 0.95 . The modal transformation matrix for the soil model, $\Phi_{d}$, was determined by the above method. Then the calculation matrices for the reduced soil FEM were determined using (2).

The equipment-structure-nonlinear and equipmentstructure-reduced soil models were elaborated, and the calculation results from the two models were compared to evaluate the modal reduction feasibility. The translational acceleration at the center of the foundation was used as the evaluation criterion. The ES subsystem can be reduced to a 5-DOF computational model, and the corresponding calculation matrices can be derived from the design parameters of the subsystem [20]. The design parameters imply that the each of the lower three stories of the structure weighed $1,700 \mathrm{~kg}$, that is, $m_{1}=m_{2}=m_{3}=1,700 \mathrm{~kg}$, 
TABLE 2: Characteristic parameters of the soil.

\begin{tabular}{lccc}
\hline Order of the Ritz vector & Order of complete mode & Frequency $(\mathrm{Hz})$ & Mass participation factor \\
\hline 1 & 1 & 1.83 & 0.59 \\
2 & 6 & 3.72 & 0.075 \\
3 & 11 & 5.08 & 0.036 \\
$\ldots$ & $\ldots$ & $\ldots$ & $\ldots$ \\
60 & 495 & 29.43 & $6.12 \times 10^{-4}$ \\
\hline Sum & & & 0.95 \\
\hline
\end{tabular}

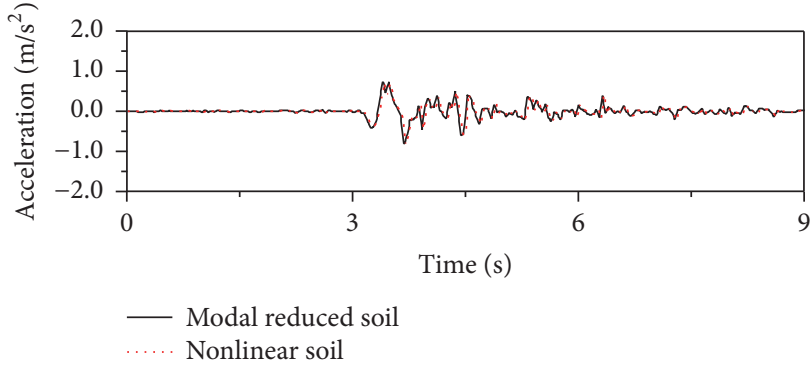

(a) Minor earthquake

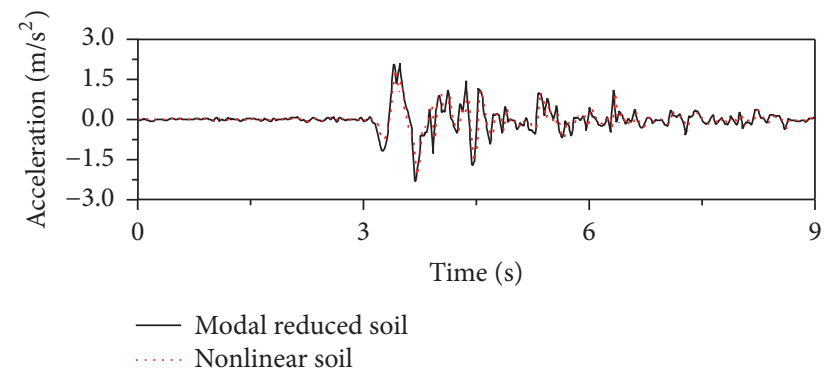

(b) Medium earthquake

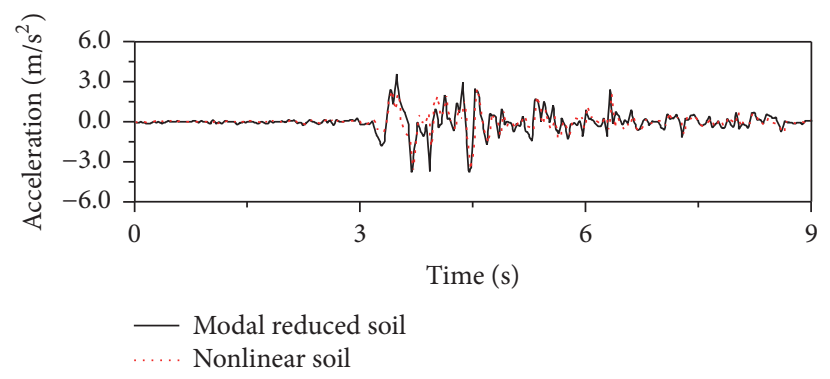

(c) Major earthquake

FIgURE 6: Comparison of the foundation center's acceleration responses produced by the two computational models.

and the mass of fourth story, $m_{4}$, was $1,540 \mathrm{~kg}$. Based on this, the stiffness $(k)$ and damping coefficients $(c)$ of these stories were calculated: the 1st story: $k_{1}=2,423,080 \mathrm{~N} / \mathrm{m}$, $c_{1}=4,463 \mathrm{~N} /(\mathrm{m} / \mathrm{s})$; the $2-4$ th stories: $k_{2}=k_{3}=k_{4}=$ $3,833,120 \mathrm{~N} / \mathrm{m}, c_{2}=c_{3}=c_{4}=7,061 \mathrm{~N} /(\mathrm{m} / \mathrm{s})$. The mass, stiffness, and damping coefficients of the equipment are as follows: $m_{5}=90 \mathrm{~kg}, k_{5}=93,046 \mathrm{~N} / \mathrm{m}$, and $c_{5}=42 \mathrm{~N} /(\mathrm{m} / \mathrm{s})$. The design parameters of the nonlinear and reduced soil models are presented in Tables 1 and 2, respectively. Based on the design parameters, an equipment-structurenonlinear soil model was constructed via the ANSYS, and an equipment-structure-reduced one was created in MATLAB using (5). Then the equation of motion was solved via the Newmark- $\beta$ direct integration method.

Taking the TianJin ground motion during the minor, medium, and major earthquakes stages as an example, the acceleration responses at the foundation center were calculated by the two computational models and depicted in Figure 6 . The foundation center peak acceleration during the minor, medium, and major earthquakes calculated by the reduced soil model exceeded those predicted by the nonlinear one by $2.4 \%, 3.7 \%$, and $11.5 \%$, respectively. The acceleration evolutions derived by the two models were consistent for the minor and medium earthquakes but differed dramatically in case of a major earthquake. This was primarily because the soil behavior was linear or exhibited a small nonlinear deformation during the minor and medium earthquakes and thus could be quite accurately described by the reduced model, while during a major earthquake the soil underwent significant nonlinear deformation, which was not taken into account by the reduced model. This proves that the reduced soil model is not applicable to the case of a major earthquake. Therefore, the study adopted the reduced soil model for the minor and medium (up to 8th degree) earthquakes in the RTDSST testing, in order to ensure the testing feasibility.

In this way, the modal reduction can effectively reduce the number of DOFs of the soil model, while ensuring the accuracy of calculations, so that adopting the reduced soil model as the numerical substructure in the analysis will provide a more efficient calculation of the numerical substructure.

3.3. Test Procedure. Figure 7 shows the composition of the RTDSST test system. The test procedure involves three major steps: (1) constructing a real-time test system; (2) designing substructure controllers; and (3) calculating the numerical 


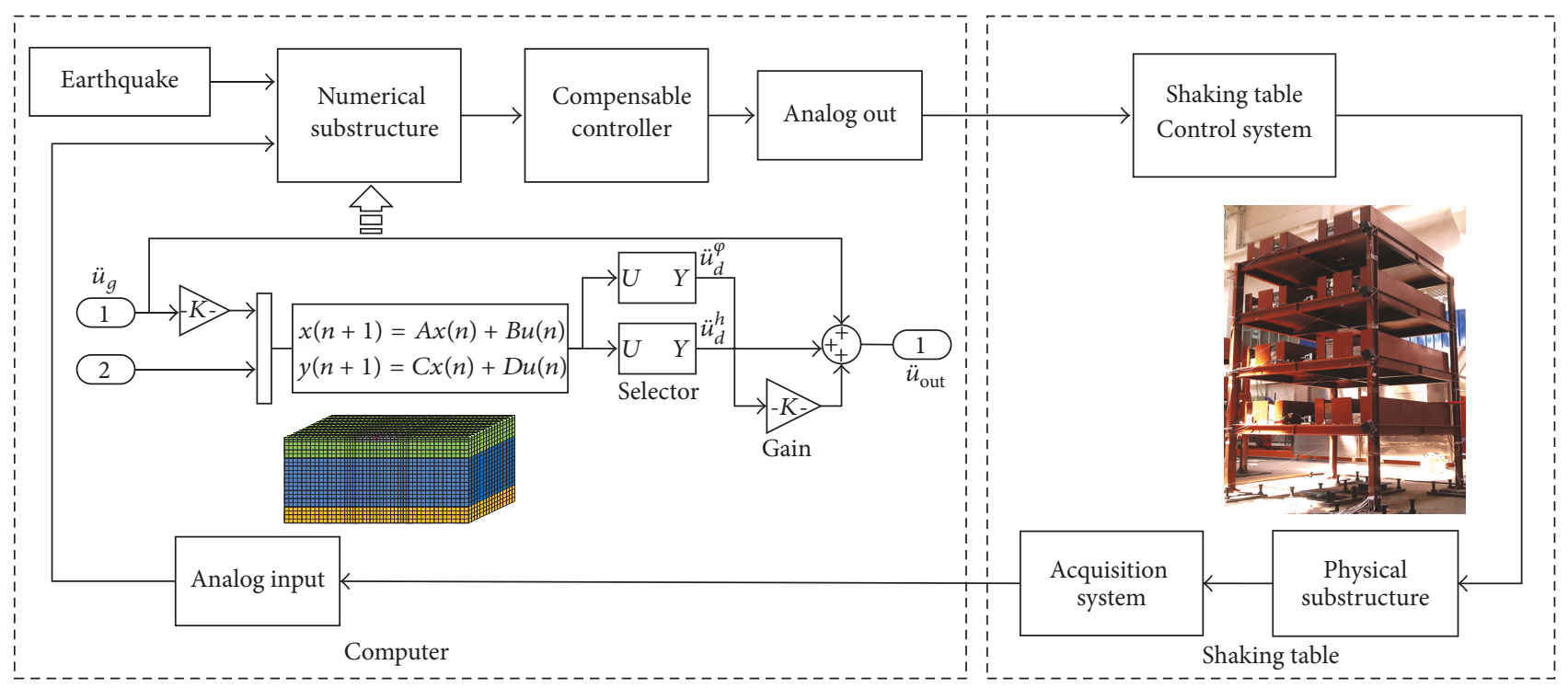

FIGURE 7: Schematic diagram of the experimental system.

substructure. A specialized computer for engineering control was used as the server in the calculation of the numerical substructure, whereas the SIMULINK simulation software was implemented for use in the substructure controller design and calculation of the numerical substructure. With the SIMULINK Desktop Real-Time [21], the test module can be compiled into a $\mathrm{C}$ code that is executable in a real-time environment. Then a computer equipped with a compiler and an I/O device can act as a real-time system and communicate with the external equipment via the I/O device. In this study, a closed-loop real-time substructure test system was set up using this method, which offered a cost-effective data communication during the real-time substructure testing process.

The dynamic response of the numerical substructure was calculated using the SIMULINK state equations. The calculation modules are shown in Figure 7. The state variable $x$ is composed of the modal displacement and modal velocity of the soil. The input variable $u$ is composed of the external and coupling loads acting on the soil. The output variable $y$ is the physical acceleration of the soil after coordinate transformations. Then, the translational and rotational acceleration of the foundation were selected and incorporated via the selector module, and its equivalent horizontal acceleration was calculated using (9). The actual seismic excitations experienced by the experimental substructure were then determined from the acceleration and used as the driving commands for the shaking table. The matrices in the state equations, denoted by $A, B, C$, and $D$, are given in

$$
\begin{aligned}
& {[A]=\left[\begin{array}{cc}
0 & I \\
-\frac{\widetilde{k}_{d}}{\widetilde{m}_{d}} & -\frac{\widetilde{c}_{d}}{\widetilde{m}_{d}}
\end{array}\right]} \\
& {[B]=\left[\begin{array}{cc}
0 & 0 \\
\frac{\widetilde{f}_{d}}{\widetilde{m}_{d}} & \frac{\widetilde{f}_{d s}}{\widetilde{m}_{d}}
\end{array}\right]}
\end{aligned}
$$

$$
\begin{aligned}
& {[C]=\left[\begin{array}{ll}
-\frac{\Phi_{d} \tilde{k}_{d}}{\widetilde{m}_{d}} & -\frac{\Phi_{d} \widetilde{c}_{d}}{\widetilde{m}_{d}}
\end{array}\right]} \\
& {[D]=\left[\begin{array}{ll}
\frac{\Phi_{d} \tilde{f}_{d}}{\widetilde{m}_{d}} & \frac{\Phi_{d} \tilde{f}_{d s}}{\widetilde{m}_{d}}
\end{array}\right] .}
\end{aligned}
$$

To track the testing setup performance more accurately, the compensation control was implemented in the RTDSST test system. Time-delay compensation normally involves the loading equipment reduction to a time-delay system, giving a constant value to the system time delay $\tau$, and sending output test commands $\tau$ seconds in advance by different prediction approaches. This procedure can ensure a consistency of the loading system input and output signals. Various prediction methods, such as single-step prediction [22, 23] and multistep prediction [24], have been proposed and applied to the real-time substructure testing. Insofar as a shaking table has a complex dynamic behavior, its reduction to a timedelay system will not completely compensate the errors in the loading system. In the inverse dynamics compensation method of the shaking table [25], the relationship between the input signal $r$ and output signal $y$ can be expressed as $y=C(s) G(s t) r$. If an accurate mathematical model of the shaking table is constructed and an inverse solution is derived from it, then $C(s) G(s t)=1$, and the amplitude and phase errors in the loading system would be eliminated. However, the complex dynamic behavior of the testing setup, combined with the influence of some external factors, often hinders the achievement of desired compensation effect by this method.

Tagawa et al. introduced a virtual object control concept and proposed an inverse dynamics compensation via the simulation scheme [26]. Further studies [27, 28] have corroborated that this scheme application allowed one to avoid interference from noise and other interfering signals from the external sources and verified its effectiveness through 


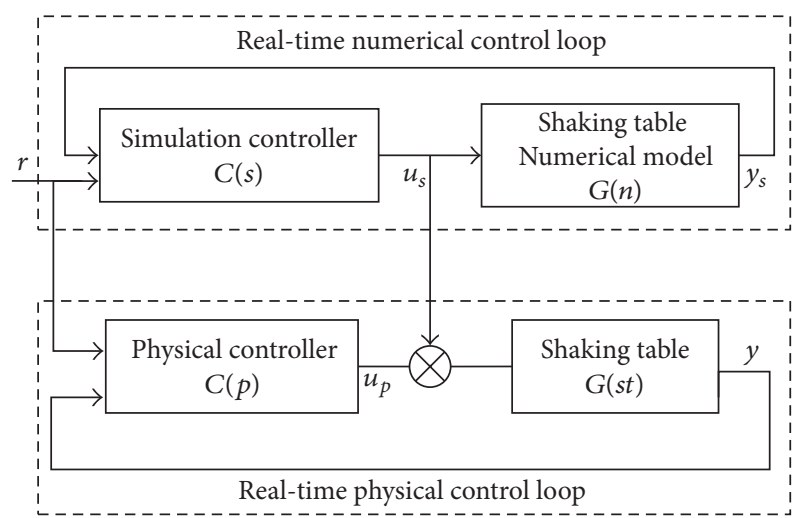

FIGURE 8: Principle of IDCS technique with closed-loop system.

the practical application to nonlinear control. Guo et al. [29] further explored a strategy for inverse dynamics compensation and developed a new control methodology called fullstate compensation via simulation (FSCS). Given various uncertainties, a control method combining the closed-loop control and real-time inverse dynamics compensation via simulation was applied to the shaking table control in this study. Figure 8 illustrates the principle of this method, which involves separate compensations of the shaking table predictable and unpredictable behaviors. For dynamic behavior that could be described by mathematical models, the inverse dynamics compensation via simulation was implemented. For uncertain dynamics that could not be described by mathematical models, a physical closed-loop control was adopted.

Based on the measured acceleration of shaking table and the reference signal within control computer, the dynamic characteristics of the shaking table system were calculated, while the fourth-order transfer function of the shaking table was identified using the MATLAB function "fminsearch." It can be expressed as follows:

$$
G=\frac{2.611 e 8}{s^{4}+913.6 s^{3}+9.525 e 4 s^{2}+1.245 e 7 s+2.613 e 8} .
$$

The amplitudes and phases obtained from the shaking table testing and the systematic identification results are plotted in Figure 9 to describe the shaking table dynamic behavior, where the transfer function fitted by the systematic identification is capable of describing the actual vibrational motion of the shaking table and is thus applicable to the inverse dynamics compensation of the numerically controlled part of the test system. The physical part is driven by the shaking table controller, which can further reduce the experimental error and provide more accurate tracking and control of the shaking table trajectory.

\section{Results}

The RTDSST of the ESS interaction system was carried out using the constructed testing platform established in Section 3.3. Ground motions with degree VIII intensity were input to observe the system responses during minor and
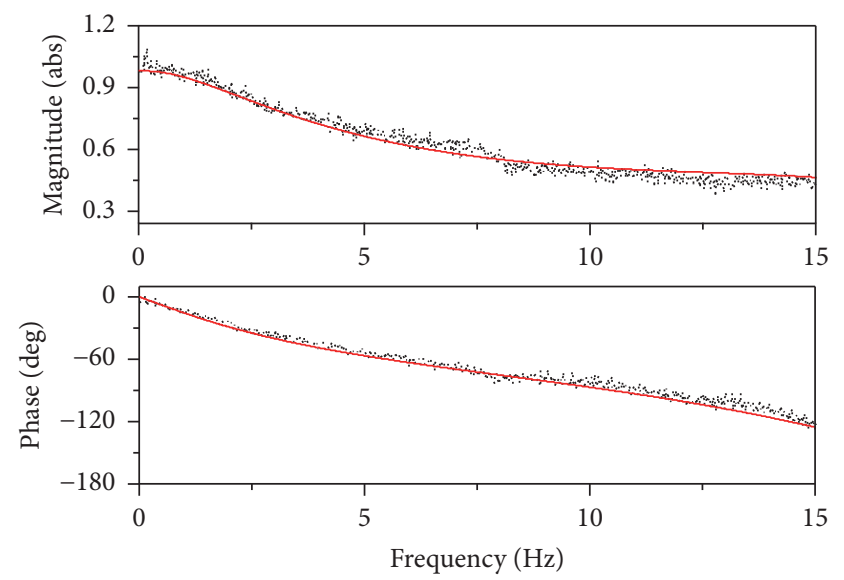

-.... Experiment

- Approximation

Figure 9: Comparison of the experimental and approximated transfer functions of shaking table.

medium earthquakes. The equipment top acceleration was used as an indicator to assess the reliability of the proposed RTDSST testing method. The results from the RTDSST testing were compared with those calculated by the integrated numerical calculation. To evaluate the soil effect on seismic responses of the equipment and structure, the shaking table tests on the ESS system and ES subsystem were performed, whereas ground motions were directly applied to the latter.

4.1. Experimental Verification. The validity of the proposed RTDSST testing method was verified by a comparison of the experimental and numerical acceleration of the equipment top. The numerical acceleration was calculated by the equipment-structure-reduced soil model implemented in MATLAB. The comparison between the experimental acceleration from the RTDSST testing and the numerical results from the integrated numerical calculation is presented in Figure 10. The comparative analysis between experimental and the numerical accelleration responses of the equipment top reveals that, during a minor earthquake with degree VIII intensity, the experimental peak acceleration of the equipment top in response to the El Centro, TianJin, and PerSon ground motions was decreased by $6.0 \%, 4.5 \%$, and $3.8 \%$, respectively, as compared to the corresponding numerical values. The equipment top acceleration evolutions obtained by the two methods exhibited a close correlation for the three ground motions. The satisfactory overall accuracy of the testing proves that the proposed RTDSST testing method is reliable and valid. The deviations between the experimental and numerical results can be attributed to the limited performance of the shaking table controller and the differences between the computational model and the actual model of the ES subsystem.

4.2. Variation Characteristics. Before the earthquakesimulating excitation test, a sine sweep test was conducted separately for the equipment and structure fixed on the shaking table, to investigate their vibration characteristics. 


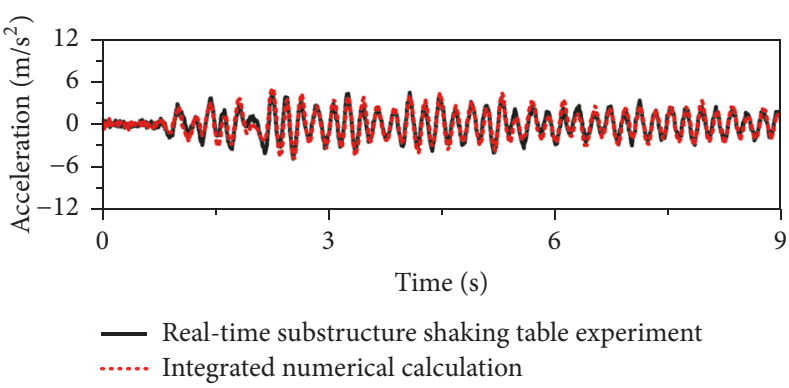

(a) El Centro

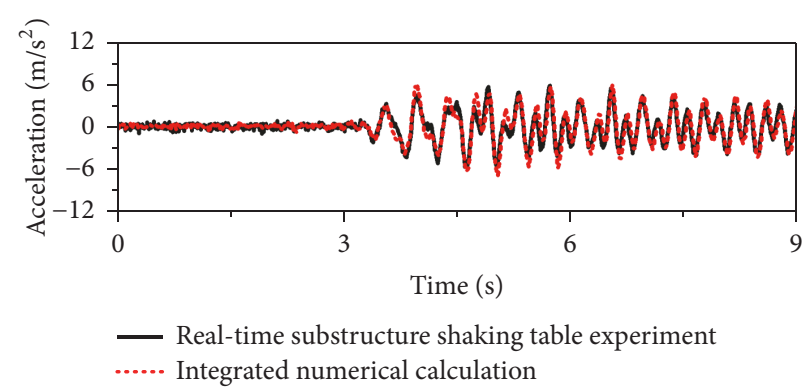

(b) TianJin

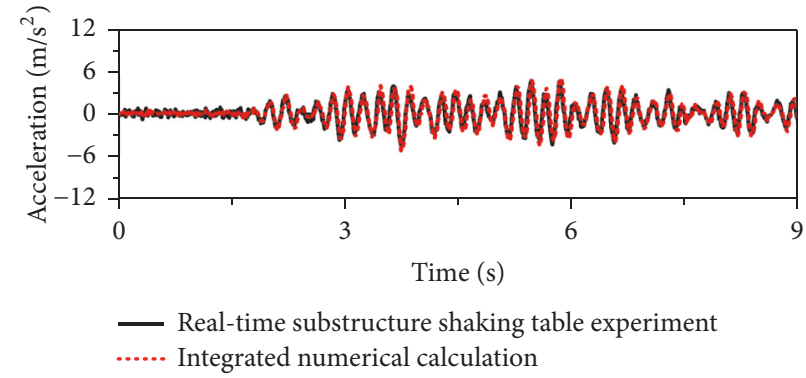

(c) PerSon

FIGURE 10: Experimental and numerical acceleration responses of the equipment top.

The sine sweep signals with frequencies from $0 \mathrm{~Hz}$ to $40 \mathrm{~Hz}$ were used to excite the shaking table. Then acceleration responses were measured at different locations of the equipment and structure. Next, the fast Fourier transform (FFT) was applied to the acceleration signals. The fundamental frequencies of the equipment and structure were found to be $5.12 \mathrm{~Hz}$ and $2.38 \mathrm{~Hz}$, respectively. After that, the equipment was connected to the structure to form an ES subsystem, which was then mounted on the shaking table. The results of sine sweep for the equipment top indicated that the initial first- and second-order frequencies of the subsystem were $2.35 \mathrm{~Hz}$ and $4.97 \mathrm{~Hz}$, respectively. The former was close to the basic structure frequency, and the latter approached the basic equipment frequency.

After the minor and medium earthquake stages of the RTDSST test were completed, sine sweep was performed for the ES subsystem. The vibration characteristics of the ES subsystem were obtained through the FTT of the acceleration signals from the equipment top and plotted in Figure 11. As seen from Figure 11, after the minor earthquake stage was finished, the first- and second-order frequencies of the subsystem did not differ from its natural ones. When the medium earthquake-simulating stage was over, the subsystem first- and second-order frequencies were $2.28 \mathrm{~Hz}$ and $4.69 \mathrm{~Hz}$, respectively, which indicated their reduction by $3.0 \%$ and $5.6 \%$, respectively, from their corresponding initial values. These results strongly suggest that the ES subsystem entered into the nonlinear state in the medium earthquake stage but fail to specify in which particular cases the plastic deformation of the equipment or structure took place.

4.3. Strain Response. Strain gauges were mounted to the equipment and structure bottom parts to measure their strain responses. The variations in peak strain can reflect the occurrence of deformation of the equipment or structure. The strain measurements of the equipment and structure indicated that the equipment behavior was linear for the El Centro and PerSon ground motions at minor and medium earthquake intensities, as well as for the Tianjin ground motion at the minor earthquake intensity. During the medium earthquake stage of the Tianjin ground motion, the equipment bottom in the ESS system and the ES subsystem exhibited peak strain values of $2,204 \mu \varepsilon$ and $2,392 \mu \varepsilon$, respectively, while the maximal peak strain of the structure bottom in the ESS system and the ES subsystem reached 1,856 $\mu \varepsilon$ and $2,081 \mu \varepsilon$, respectively, which exceeded the elastic limits of the equipment and structure. This implies that the hardware and structure both exhibited a nonlinear behavior at this stage. The strain measurements also revealed that the states of the equipment and structure varied with ground motion types. During the medium earthquake, both the equipment and structure behaviors were nonlinear for the TianJin ground motion and linear for other cases.

4.4. Acceleration Response. Variations in the acceleration responses of the equipment and structure under different ground motions were analyzed to assess the soil effect on their seismic performance. Tables 3 and 4 summarize the peak acceleration measured at the tops of the equipment and structure in the ES subsystem and the ESS system, respectively. A comparative analysis shows that, due to the soil effect, the equipment top peak acceleration under the El Centro, TianJin, and PerSon ground motions decreased by $9.2 \%, 18.3 \%$, and $12.0 \%$, respectively, during the minor earthquake stage. Meanwhile, the structure top peak acceleration dropped by $15.1 \%, 23.6 \%$, and $10.9 \%$, respectively. During the medium 


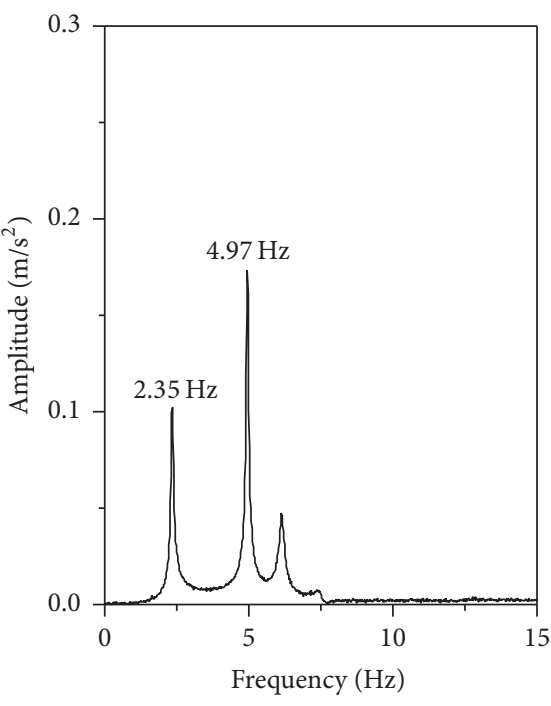

(a) Minor earthquake

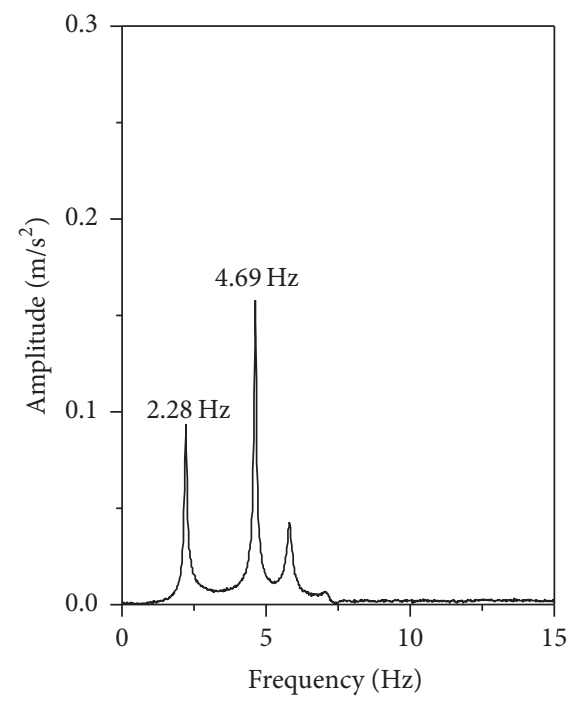

(b) Medium earthquake

FIGURE 11: Variation characteristics of the ES subsystem during the minor and medium earthquakes.

TABLE 3: Equipment top peak accelerations.

\begin{tabular}{lcc}
\hline Earthquake stage & ES subsystem $\left(\mathrm{m} / \mathrm{s}^{2}\right)$ & ESS system $\left(\mathrm{m} / \mathrm{s}^{2}\right)$ \\
\hline Minor & & \\
El Centro & 6.19 & 5.62 \\
TianJin & 7.33 & 5.99 \\
PerSon & 6.48 & 5.70 \\
Medium & & \\
El Centro & 15.92 & 14.42 \\
TianJin & 16.53 & 15.92 \\
PerSon & 17.13 & 15.16 \\
\hline
\end{tabular}

TABLE 4: Structure top peak accelerations.

\begin{tabular}{lcc}
\hline Earthquake stage & ES subsystem $\left(\mathrm{m} / \mathrm{s}^{2}\right)$ & ESS system $\left(\mathrm{m} / \mathrm{s}^{2}\right)$ \\
\hline Minor & & \\
El Centro & 3.50 & 2.97 \\
TianJin & 4.83 & 3.69 \\
PerSon & 1.93 & 1.72 \\
Medium & & \\
El Centro & 8.76 & 7.49 \\
TianJin & 9.65 & 8.03 \\
PerSon & 5.26 & 4.66 \\
\hline
\end{tabular}

earthquake stage, the interaction between the ES subsystem and the soil resulted in the reduction of equipment top peak acceleration under the El Centro, TianJin, and PerSon ground motions by $9.4 \%, 3.7 \%$, and $11.5 \%$, respectively. The corresponding reductions of the structure top acceleration were $14.5 \%, 16.8 \%$, and $11.4 \%$, respectively.

The comparative analysis about the acceleration responses of the equipment and structure proves that, in the presence of the soil, the ESS subsystem experienced a reduction of the equipment top and structure top acceleration responses, and the rate of this reduction closely correlated with the type of ground motion and seismic intensity. For instance, under the TianJin ground motion, the equipment top acceleration decreased by $18.3 \%$ during the minor earthquake, which was much higher than the reduction by $3.7 \%$, which was observed during the medium earthquake simulation.

4.5. Shear Forces. The shear forces of the ES subsystem under different conditions can be calculated from the acceleration responses. Figure 12 compares the shear force responses of the ES subsystem and the ESS system during the minor earthquake: the ESS system shear forces are much lower than those of the ES subsystem. In case of the soil effect account, the peak values of the ESS system shear force under the El Centro, TianJin, and PerSon ground motions decreased by $20.3 \%, 17.7 \%$, and $28.7 \%$, respectively. The shear force responses of the ES subsystem and the ESS system during the medium earthquake are illustrated in Figure 13. Under the El Centro, Tianjin, and PerSon ground motions, the shear force peak values of the ESS system dropped by $20.5 \%, 4.3 \%$, and $28.2 \%$, respectively, as compared to the ES subsystem.

The comparison of shear force responses between ES subsystem and ESS system shows that the shear forces of the ESS system tended to decrease under the soil effect because the interaction with the soil mitigated the seismic responses of the equipment and structure and thereby reduced the shear forces of the subsystem. However, under the TianJin ground motion, the rate of shear force reduction varied significantly between the two earthquake stages. This can be attributed to the fact that the ES subsystem behavior became nonlinear at the medium earthquake stage.

4.6. Displacement Response. The displacement responses of all stories of the equipment and structure were measured. Figure 14 shows the peak displacements of different stories 

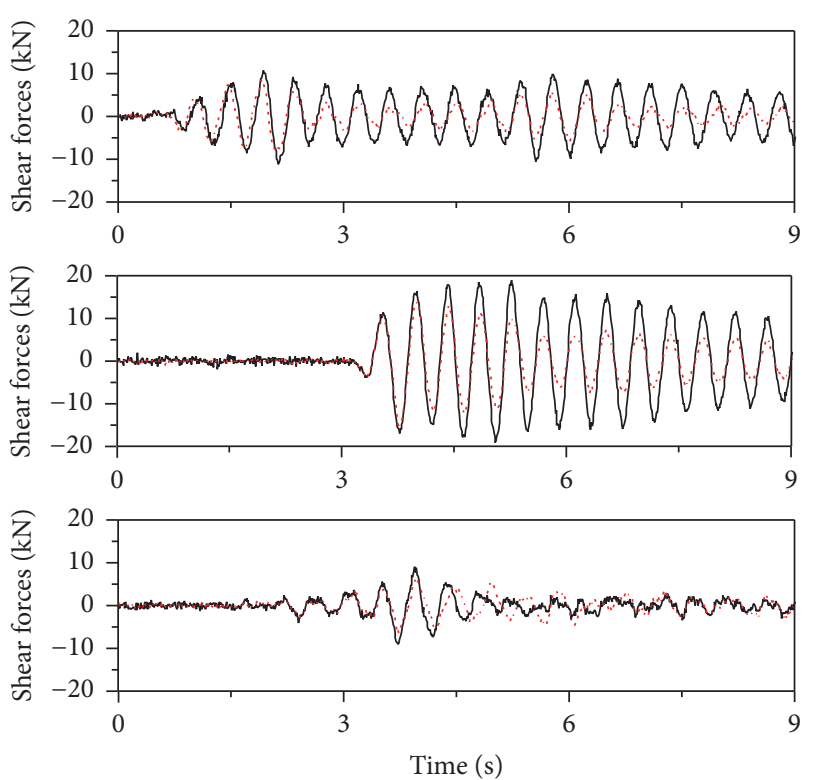

- Equipment-structure subsystem Equipment-structure-soil system

FIGURE 12: Shear force responses of different systems during the minor earthquake.
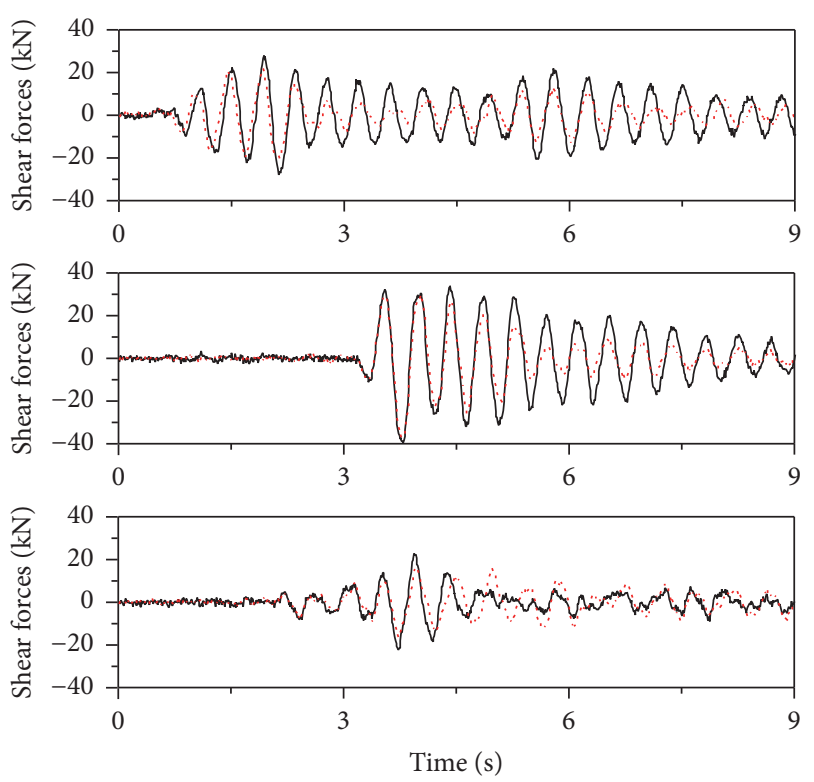

- Equipment-structure subsystem Equipment-structure-soil system

FIGURE 13: Shear force responses of different systems during the medium earthquake.

of the ES subsystem and the ESS system during the minor earthquake. The measurements from the first through fourth stories represent the peak displacements of the four stories of the structure, and those from the fifth story are the equipment top peak displacements. One can see from Figure 14 that the peak displacements of each story of the ESS system are much smaller than the corresponding values of the ES subsystem.
Under the El Centro, TianJin, and PerSon ground motions, the peak displacements of the equipment top in the ESS system decreased by $19.1 \%, 26.5 \%$, and $19.0 \%$, respectively, as compared to those measured in the ES system. In case of the soil effect account, the respective structure top peak displacements exhibited the reduction by $16.2 \%, 20.1 \%$, and $25.5 \%$. The peak displacement responses during the medium earthquake are shown in Figure 15. In case of the soil effect account, the peak displacements of the equipment top under the three types of ground motions dropped by $18.6 \%, 8.1 \%$, and $18.9 \%$, respectively, while those of the structure top decreased by $15.9 \%, 9.5 \%$, and $26.3 \%$, respectively.

As described in this section about the displacement responses of the equipment and structure, the peak displacements of the equipment and structure were substantially reduced due to the soil effect, mainly because the soil damping increased the energy dissipation. During a minor earthquake, the maximum drop of the equipment peak displacement occurred under the TianJin ground motion, while the maximum decrease in the structure peak displacement occurred under the PerSon ground motion. Under the TianJin ground motion, the tops of the two components underwent greater variations in peak displacement during a medium earthquake than during a minor one, primarily due to nonlinear behavior of both the equipment and structure during a medium earthquake. This implies that, as the seismic intensity increased, the self-deformation states of the equipment or structure tended to exert a significant influence on their seismic responses.

In this study, the integrated testing of an ESS interaction system was successfully implemented using a realtime shaking table testing method based on the branch substructure method. The proposed method adopts the equipment-structure subsystem as the experimental substructure and effectively combines the numerical calculation with experimental testing. Moreover, the modal reduction of the numerical model of soil improved the efficiency of the real-time substructure testing with no deterioration of the calculation accuracy. Therefore, the proposed method is quite precise and applicable to practical experiments, which makes it quite lucrative and instrumental in the evaluation of seismic performance of various interactive systems.

\section{Conclusions}

In this study, the branch substructure method was used to derive the equation of motion of an equipment-structuresoil interaction system, which was then subjected to the RTDSST test. The results obtained make it possible to draw the following conclusions:

(1) After rearrangement, the formulated equation of motion was successfully applied to the RTDSST testing of the ESS interaction system. This testing method has a clear concept and is convenient to use.

(2) The foundation rotational acceleration was converted to the equivalent horizontal acceleration applied to the experimental substructure based on the principle of equivalence. In this way, the rotation of foundation 


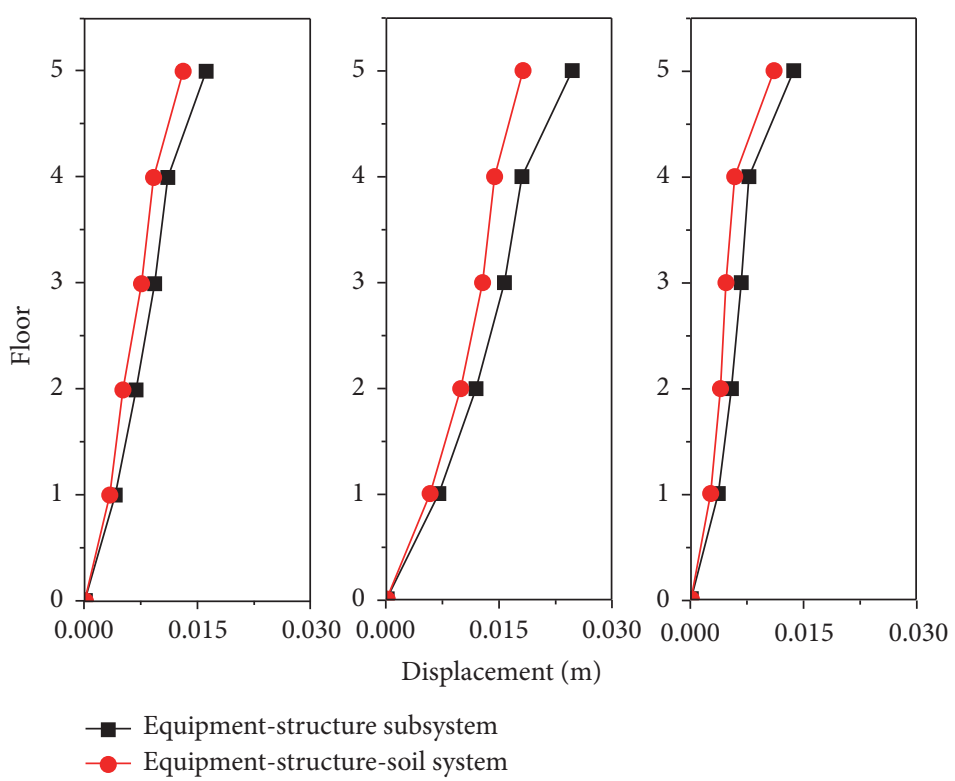

FIGURE 14: Peak displacement responses during the minor earthquake.
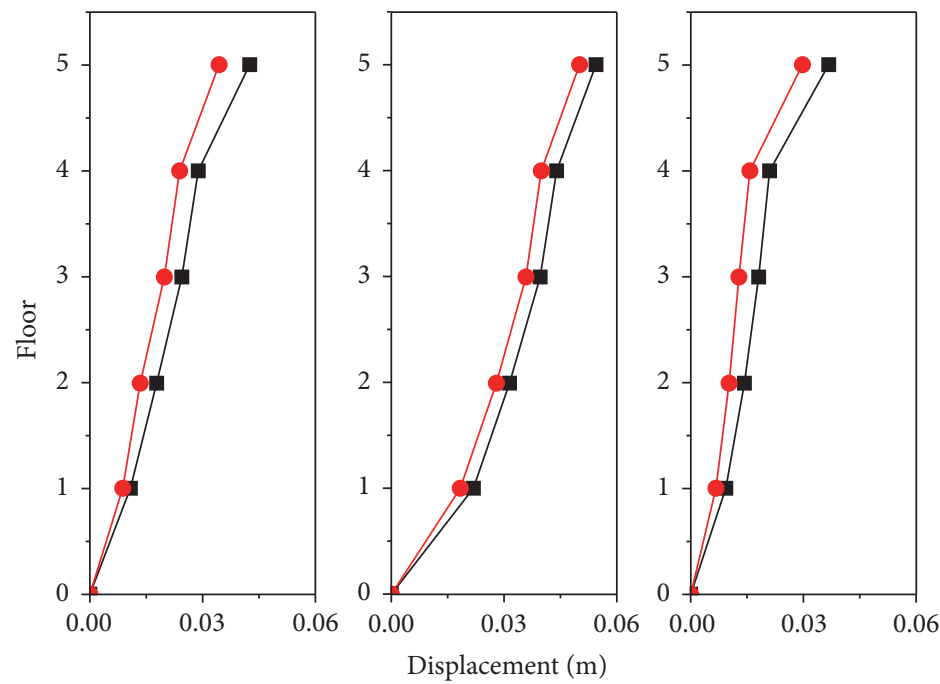

Equipment-structure subsystem

Equipment-structure-soil system

FIgURE 15: Peak displacement responses during the medium earthquake.

was taken into account in the translational shaking table testing and, thus, the accuracy of test results was improved.

(3) A comparison of the experimental and numerical results revealed their good fit, which confirms the reliability and validity of the RTDSST testing method.

(4) The soil effect on the equipment and structure is manifested by the overall reduction of their seismic responses. With an increase in the seismic intensity, the self-deformation states of the equipment and structure increasingly affect their seismic responses.

The modal reduction involved in the RTDSST method, which is proposed in this study, quite accurately accounts for the effect of soil with a linear behavior, but further research is needed to investigate more complex cases of nonlinear soil behavior.

\section{Conflicts of Interest}

The authors declare that there are no conflicts of interest regarding the publication of this paper.

\section{Acknowledgments}

This work was financially supported by the National Science Foundation Project (Research Project no. 51478312), National Youth Science Foundation Project (Research Project no. 
51208356), and the National Science Foundation Project (Research Project no. 51278335), China.

\section{References}

[1] J. Li, H. Chen, and Z. S. Sun, "Shaking table tests on spatial structure-equipment model systems," Engineering Mechanics, vol. 20, no. 1, pp. 157-161, 2003.

[2] E. Pantoli, X. Wang, M. Chen, T. Hutchinson, B. Meacham, and H. J. Park, "Shake table testing of a full-scale five-story building: performance of the major nonstructural componentsegress and facades," in Proceedings of the Structures Congress 2013: Bridging Your Passion with Your Profession, pp. 1447-1459, Pittsburgh, Pennsylvania, PA, USA, May 2013.

[3] G. Biondi, M. R. Massimino, and M. Maugeri, "Experimental study in the shaking table of the input motion characteristics in the dynamic SSI of a SDOF model," Bulletin of Earthquake Engineering, vol. 13, no. 6, pp. 1835-1869, 2015.

[4] X. Gao, X.-Z. Ling, L. Tang, and P.-J. Xu, "Soil-pile-bridge structure interaction in liquefying ground using shake table testing," Soil Dynamics and Earthquake Engineering, vol. 31, no. 7, pp. 1009-1017, 2011.

[5] R. Paolucci, M. Shirato, and M. T. Yilmaz, "Seismic behaviour of shallow foundations: Shaking table experiments vs numerical modelling," Earthquake Engineering \& Structural Dynamics, vol. 37, no. 4, pp. 577-595, 2008.

[6] G. Abate, M. R. Massimino, and M. Maugeri, "Numerical modelling of centrifuge tests on tunnel-soil systems," Bulletin of Earthquake Engineering, vol. 13, no. 7, pp. 1-25, 2015.

[7] R. Figini, R. Paolucci, and C. Chatzigogos, "A macro-element model for non-linear soil-shallow foundation-structure interaction under seismic loads: Theoretical development and experimental validation on large scale tests," Earthquake Engineering \& Structural Dynamics, vol. 41, no. 3, pp. 475-493, 2012.

[8] P. Galvín and A. Romero, "A MATLAB toolbox for soilstructure interaction analysis with finite and boundary elements," Soil Dynamics and Earthquake Engineering, vol. 57, pp. 10-14, 2014.

[9] X. L. Jiang and B. W. Xu, "Analysis of shaking table test of large-scale soil-pile-complex structure interaction," China Civil Engineering Journal, vol. 43, no. 10, pp. 98-105, 2010.

[10] X. Jiang and Y. Li, "Shaking table test of soil-pile-eccentric structure interaction system," Journal of Building Structures, vol. 31, no. 8, pp. 106-111, 2010.

[11] M. Nakashima, H. Kato, and E. Takaoka, "Development of real-time pseudo dynamic testing," Earthquake Engineering \& Structural Dynamics, vol. 21, no. 1, pp. 79-92, 1992.

[12] Q. Wang, J.-T. Wang, F. Jin, F.-D. Chi, and C.-H. Zhang, "Real-time dynamic hybrid testing for soil-structure interaction analysis," Soil Dynamics and Earthquake Engineering, vol. 31, no. 12, pp. 1690-1702, 2011.

[13] X.-Y. Yan, Z.-X. Li, Q. Han, and X.-L. Du, "Shaking tables test on a long-span rigid-framed bridge considering soil-structure interaction," Engineering Mechanics, vol. 31, no. 2, pp. 58-65, 2014.

[14] Z. Tang, H. Ma, J. Guo, and Z. Li, "Effect of Soil-Structure Interaction on Seismic Performance of Long-Span Bridge Tested by Dynamic Substructuring Method," Shock and Vibration, vol. 2017, Article ID 4358081, 12 pages, 2017.

[15] X. L. Jiang and S. C. Yan, "Two step method of the branched modal and its use for liquid-structure-pile-soil interaction system," Journal of Vibration Engineering, vol. 7, no. 4, pp. 346351, 1994.

[16] F. Wang and X. L. Jiang, "Analysis of soil-structure interaction system based on mixed branch mode and constrained mode two-step method," Journal of Earthquake Engineering and Engineering Vibration, vol. 30, no. 4, pp. 24-30, 2010.

[17] F. Wang, Dynamic substructure method for soil-structuremultistory buildings interaction system, Tianjin University, 2010.

[18] X. L. Jiang and H. S. Zhang, "Mixed constraint modal method for analysis of soil-structure system under complex conditions," Journal of Hohai university (Natural sciences), vol. 44, no. 4, pp. 283-290, 2016.

[19] Y. Li and X. Jiang, "Parametric analysis of eccentric structuresoil interaction system based on branch mode decoupling method," Soil Dynamics and Earthquake Engineering, vol. 48, pp. 63-70, 2013.

[20] S.-K. Lee, E. C. Park, K.-W. Min, and J.-H. Park, "Real-time substructuring technique for the shaking table test of upper substructures," Engineering Structures, vol. 29, no. 9, pp. 22192232, 2007.

[21] R. Grepl, "Real-time control prototyping in MATLAB/simulink: review of tools for research and education in mechatronics," in Proceedings of the IEEE International Conference on Mechatronics (ICM 2011), pp. 881-886, Turkey, April 2011.

[22] T. Horiuchi, M. Inoue, T. Konno, and Y. Namita, "Real-time hybrid experimental system with actuator delay compensation and its application to a piping system with energy absorber," Earthquake Engineering \& Structural Dynamics, vol. 28, no. 10, pp. 1121-1141, 1999.

[23] F.-D. Chi, J.-T. Wang, Q. Wang, and F. Jin, "Delay-dependent stability analysis of MDOF real-time dynamic hybrid testing considering compensation," Engineering Mechanics, vol. 28, no. 4, pp. 200-207, 2011.

[24] M. I. Wallace, D. J. Wagg, and S. A. Neild, "An adaptive polynomial based forward prediction algorithm for multi-actuator real-time dynamic substructuring," Proceedings of the Royal Society A Mathematical, Physical and Engineering Sciences, vol. 461, no. 2064, pp. 3807-3826, 2005.

[25] J. E. Carrion, B. F. Spencer Jr., and B. M. Phillips, "Realtime hybrid simulation for structural control performance assessment," Earthquake Engineering and Engineering Vibration, vol. 8, no. 4, pp. 481-492, 2010.

[26] Y. Tagawa, J.-Y. Tu, and D. P. Stoten, "Inverse dynamics compensation via 'simulation of feedback control systems," Proceedings of the Institution of Mechanical Engineers, Part I: Journal of Systems and Control Engineering, vol. 225, no. 1, pp. 137-153, 2011.

[27] X. Ji, K. Kajiwara, T. Nagae, R. Enokida, and M. Nakashima, “A substructure shaking table test for reproduction of earthquake responses of high-rise buildings," Earthquake Engineering \& Structural Dynamics, vol. 38, no. 12, pp. 1381-1399, 2009.

[28] J. Y. Tu, P. Y. Lin, D. P. Stoten, and G. Li, “Testing of dynamically substructured, base-isolated systems using adaptive control techniques," Earthquake Engineering \& Structural Dynamics, vol. 39, no. 6, pp. 661-681, 2010.

[29] J. Guo, Z. Tang, S. Chen, and Z. Li, "Control strategy for the substructuring testing systems to simulate soil-structure interaction," Smart Structures and Systems, vol. 18, no. 6, pp. 1169-1188, 2016. 


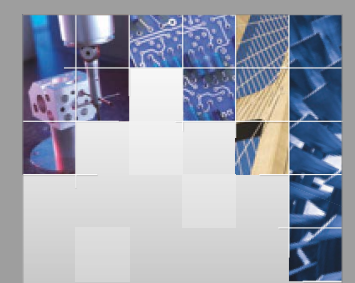

\section{Enfincering}
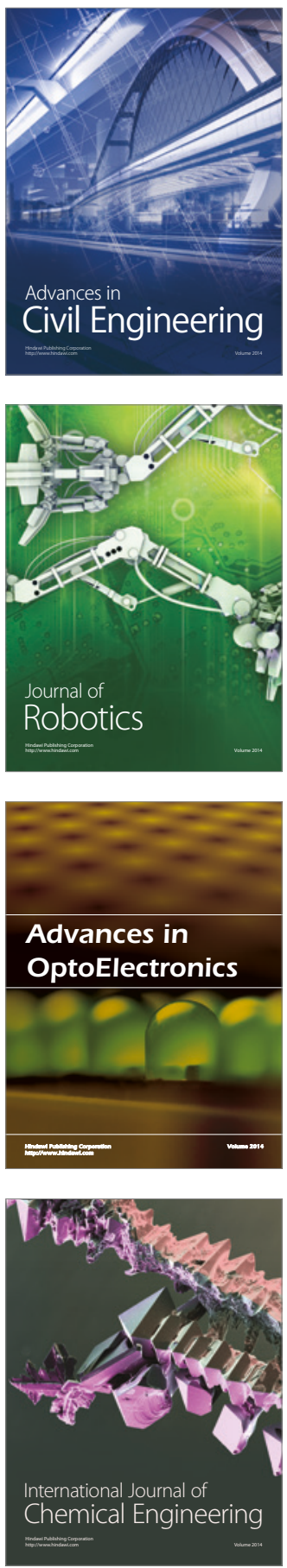

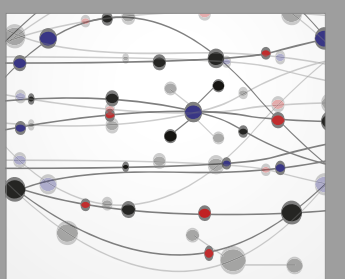

The Scientific World Journal

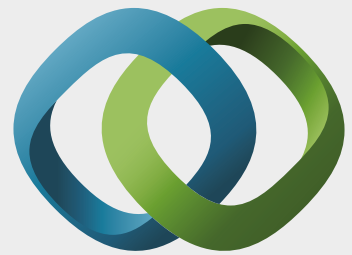

\section{Hindawi}

Submit your manuscripts at

https://www.hindawi.com
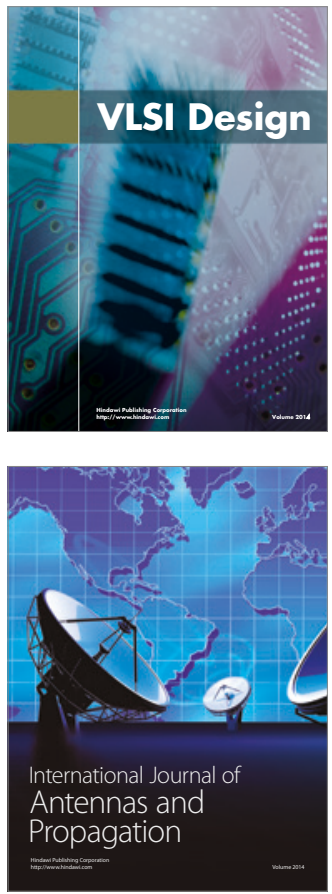

\section{Rotating}

Machinery
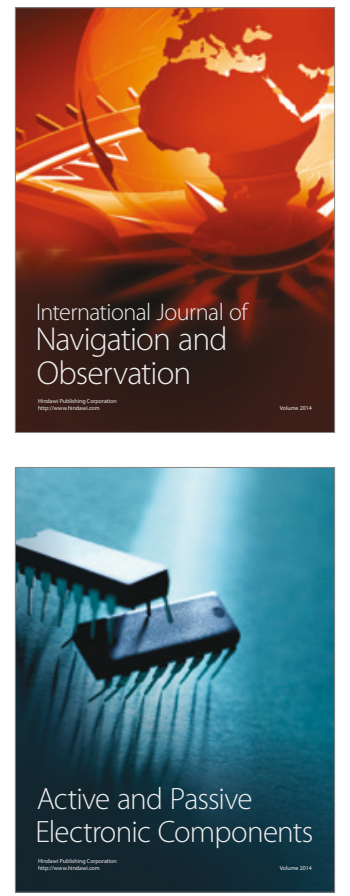
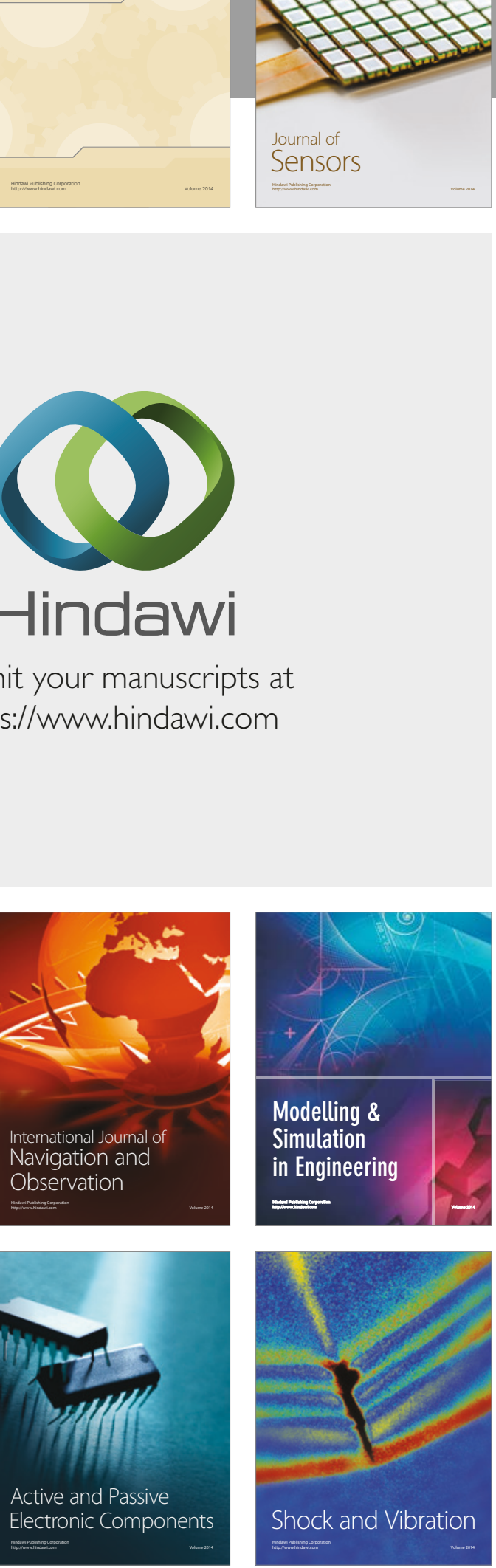
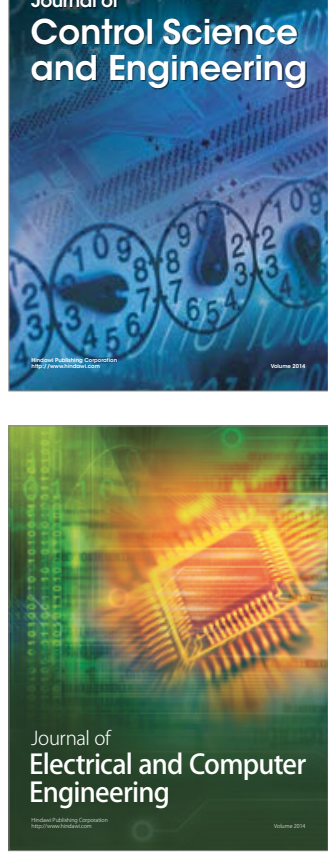

Distributed

Journal of

Control Science

and Engineering
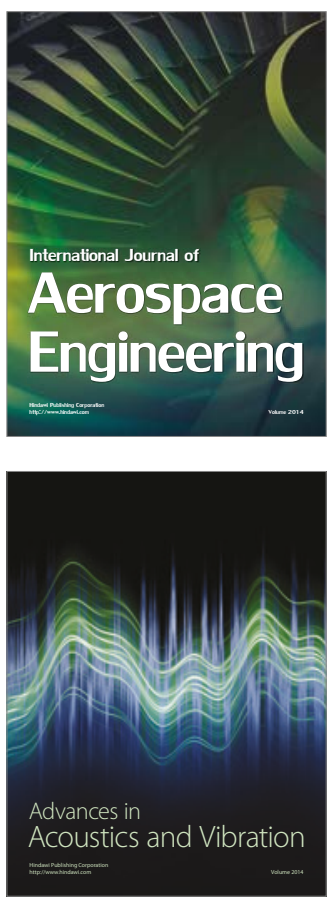

Sensor Networks 